epigenetic control of autophagy might thus be a novel approach to ameliorate fibrotic tissue remodeling.

References:

[1] Wynn, T.A. Cellular and molecular mechanisms of fibrosis. J Pathol 214, 199210 (2008).

[2] Distler, J.H., et al. Review: Frontiers of Antifibrotic Therapy in Systemic Sclerosis. Arthritis \& rheumatology (Hoboken, N.J.) 69, 257-267 (2017).

[3] Gyorfi, A.H., Matei, A.E. \& Distler, J.H.W. Targeting TGF-beta signaling for the treatment of fibrosis. Matrix biology: journal of the International Society for Matrix Biology 68-69, 8-27 (2018).

[4] Wang, C.W. \& Klionsky, D.J. The molecular mechanism of autophagy. Mol Med 9, 65-76 (2003)

[5] Hernandez-Gea, V., et al. Autophagy releases lipid that promotes fibrogenesis by activated hepatic stellate cells in mice and in human tissues. Gastroenterology 142, 938-946 (2012)

Disclosure of Interests: Ariella Zehender: None declared, Neng Yu Lin: None declared, Yi-Nan Li: None declared, Andrea-Hermina Györfi: None declared, Christina Bergmann: None declared, Andreas Ramming Grant/research support from: Pfizer, Novartis, Consultant of: Boehringer Ingelheim, Novartis, Gilead, Pfizer, Speakers bureau: Boehringer Ingelheim, Roche, Janssen, Georg Schett Speakers bureau: AbbVie, BMS, Celgene, Janssen, Eli Lilly, Novartis, Roche and UCB, Jörg Distler Grant/research support from: Boehringer Ingelheim, Consultant of: Boehringer Ingelheim, Paid instructor for: Boehringer Ingelheim, Speakers bureau: Boehringer Ingelheim

DOI: 10.1136/annrheumdis-2020-eular.3503

\section{SAT0285 VISUALISATION OF THE ACTIVE CALCIFICATION PROCESS WITH 18-F SODIUM FLUORIDE PET/CT IN LIMITED CUTANEOUS SYSTEMIC SCLEROSIS WITH CALCINOSIS CUTIS IS FEASIBLE: A PILOT STUDY}

I. M. Atzeni ${ }^{1}$, E. M. Hogervorst ${ }^{1}$, G. M. Swart ${ }^{1}$, K. De Leeuw ${ }^{2}$, M. Bij $\left.\right|^{3}$, R. Bos ${ }^{4}$, J. Westra ${ }^{2}$, G. Diercks ${ }^{5}$, H. Van Goor ${ }^{5}$, M. C. Bolling ${ }^{6}$, R. Slart ${ }^{7}$, D. J. Mulder ${ }^{1} .{ }^{1}$ University of Groningen, University Medical Center Groningen, Department of Internal Medicine, Division Vascular Medicine, Groningen, Netherlands; ${ }^{2}$ University of Groningen, University Medical Center Groningen, Department of Rheumatology \& Clinical Immunology, Groningen, Netherlands; ${ }^{3}$ Martini Hospital, Department of Rheumatology, Groningen, Netherlands; ${ }^{4}$ Medical Center of Leeuwarden, Department of Rheumatology, Leeuwarden, Netherlands; ${ }^{5}$ University of Groningen, University Medical Center Groningen, Department of Pathology \& Medical Biology, Groningen, Netherlands; ${ }^{6}$ University of Groningen, University Medical Center Groningen, Department of Dermatology, Groningen, Netherlands; ${ }^{7}$ University of Groningen, University Medical Center Groningen, Department of Nuclear Medicine \& Molecular Imaging, Groningen, Netherlands

Background: Calcinosis cutis is a major daily challenge to patients with longstanding systemic sclerosis (SSc), negatively affecting their quality of life. Unfortunately, treatment options are very limited due to lack of understanding of the pathogenetic process. Currently, calcinosis cutis is only detected at its irreversible end-stage. Early detection of calcinosis cutis could putatively allow early disease-modifying interventions and monitor treatment effects.

Objectives: The aim of the current study is to assess the feasibility of visualising "active" micro-calcifications with 18-F Sodium Fluoride (NaF) PET scanning, compared to low-dose CT in patients with clinically overt calcinosis cutis.

Methods: This was a cross-sectional, observational, pilot study. All patients met 2013 ACR/EULAR criteria for SSc. Patients underwent a whole body NaF PET/ low-dose CT scan, scanned 90 minutes post-injection. (Sub)cutaneous calcifications were described and assessed on $\mathrm{NaF}$ PET, which was compared to CT images by two independent investigators.

Results: A total of 10 female patients with limited cutaneous SSc [median age 56 years (IQR 52-66), median disease duration 17 years (8-19), PAH 10\%, ILD $20 \%$ ] were included, and compared to 10 controls [70 years (65-73)]. NaF uptake showed normal distribution throughout the skeletal bones, arterial tree, and visceral organs, which was comparable between patients and controls. Additionally, $\mathrm{NaF}$ uptake was visible in the skin of all SSc patients, but in none of the controls. Cutaneous NaF uptake largely correlated with clinical calcifications. Most common sites of cutaneous $\mathrm{NaF}$ uptake were fingers (6 patients) and knees (7 patients). Only $5 \%$ of the NaF positive lesions were not accompanied by visible calcifications on CT. Furthermore, of all calcified lesions seen on CT, $51 \%$ showed uptake on $\mathrm{NaF}$ PET. Small lesions $(<1 \mathrm{~cm})$, were generally only visible on $\mathrm{CT}$, due to lower resolution of $\mathrm{NaF}$ PET.

Conclusion: Imaging of "active" calcinosis cutis in limited cutaneous systemic sclerosis is feasible using NaF PET scanning. Most clinically overt calcifications and half of those seen on CT were positive for $\mathrm{NaF}$ uptake. Whether these "active" calcifications behave differently in terms of faster progression, clinical complaints, and infection risk, and whether these are potentially suitable for disease modifying interventions is subject to future study.

\section{Fig. 1 Clinical calcinosis cutis with corresponding images of 18F-NaF PET/CT-scan}

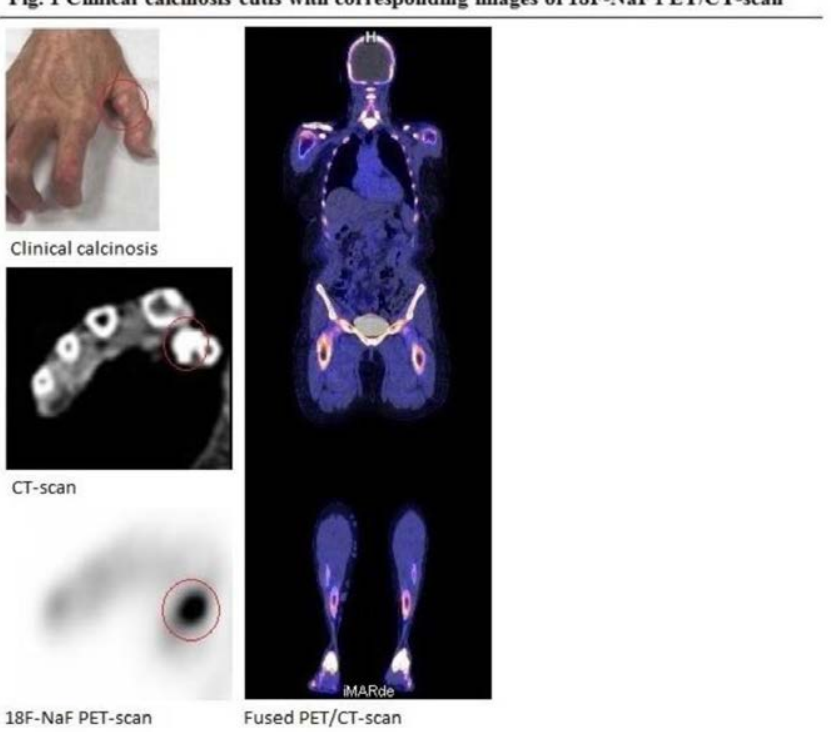

Disclosure of Interests: None declared DOI: 10.1136/annrheumdis-2020-eular.4629

\section{SAT0286 BIOLOGICAL CORRELATES OF RADIOGRAPHIC FEATURES OF INTERSTITIAL LUNG DISEASE IN SYSTEMIC SCLEROSIS: AN IN DEPTH ANALYSIS OF BRONCHOALVEOLAR PROTEINS OF SCLERODERMA LUNG STUDY I PARTICIPANTS}

E. Volkmann ${ }^{1}$, D. Tashkin ${ }^{1}$, N. Li ${ }^{1}$, G. Kim ${ }^{1}$, J. Goldin ${ }^{1}$, A. Harui ${ }^{1}$, M. Roth ${ }^{1}$.

${ }^{1}$ UCLA, Los Angeles, United States of America

Background: Systemic sclerosis-related interstitial lung disease (SSc-ILD) involves a combination of inflammation, fibrosis and vascular pathology that is typically assessed on CT imaging as a mixture of ground-glass opacification (GGO) and fibrotic changes. We hypothesized that proteins recovered from bronchoalveolar lavage (BAL) could be used to probe the underlying pathobiology associated with GGO and fibrotic changes.

Objectives: (1) To assess the relationship between 68 unique BAL proteins measured in participants of Scleroderma Lung Study (SLS) $I^{1}$ and radiographic and physiologic measures of ILD; (2) To identify inter-correlations among specific proteins to enlighten our understanding of how specific biological pathways contribute to SSc-ILD.

Methods: Bronchoscopy was performed on 144 of the 158 participants in SLS I with 103 BAL samples available for analysis. BAL was lyophilized, concentrated 10X and used in a multiplex protein analysis for 68 different cytokines chemokines and other factors. Kendall tau correlations were performed to assess the relationship between individual proteins and baseline measures of pulmonary function and quantitative CT scores for fibrosis, GGO and total ILD. Those proteins found to correlate significantly with at least 2 clinical measures of ILD were entered into a cluster analysis with inter-correlations expressed as a heatmap.

Results: Significant correlations were observed between fibrosis scores and several biologic pathways including pro-fibrotic factors (transforming growth factor beta [TGF- $\beta$ ], platelet-derived growth factor [PDGF]), proteins involved in tissue remodeling (Matrix metallopeptidase [MMP]-1,7,8,9; Hepatocyte growth factor [HGF]), and those involved in monocyte/macrophage migration and activation (Monocyte chemoattractant protein [MCP]-1,3; macrophage colony-stimulating factor [MCSF]). These same pathways correlated with the diffusing capacity for carbon monoxide (DLCO). In contrast, GGO scores correlated primarily with immune and inflammatory mediators (interleukin [IL]-5,8,13,15, IL-1 receptor antagonist and interferon gamma) with only limited overlap to proteins that related to fibrosis. Vascular endothelial growth factor (VEGF) levels were lower in patients with more extensive GGO, fibrosis and diffusion impairment, suggesting that vascular changes are a central feature of SSc-ILD. Specific proteins were highly correlated with one another in a pattern suggesting biologically-related networks (Figure) that might provide additional insight regarding disease pathogenesis. 